\title{
Long-wave spin excitations of crystalline $s-d$ models
}

\author{
Yu.Rudavsky, G.Ponedilok, L.Dorosh \\ State University "Lvivska Politechnika" \\ 12 S.Bandera St., UA-290646 Lviv-13, Ukraine
}

Received June 6, 1998

\begin{abstract}
Long-wave spin excitations in the perfect s-d model are studied. The Hamiltonian of the model includes, apart from usually treated spin-electron processes, the Heisenberg exchange interaction of atomic spins and the Coulomb interaction of conductive electrons. Hartree-Fock and Tyablikov approximations are used for closing the hierarchy of equations of motion for the double-time Green functions. The equation for the magnon excitations spectrum in this approximation is obtained.
\end{abstract}

Key words: magnon, magnetization, dispersion law

PACS: $75.30 . D s, 75.50 . K$

\section{Introduction}

The properties of metals and semiconductors which include atoms of transition or rear-earth elements are investigated within different microscopic models capacities. The $s-d$ model occupies an important place among them. This model takes into account the presence of the subsystem of magnetic moments localized on atoms of transition elements and the subsystem of band electrons. These subsystems are connected with the exchange interaction.

Theoretical investigations of the $s-d$ model focus on the collective spin excitations, named magnons. This is caused by the following reasons. Firstly, the magnon excitations (especially long-wave excitations can be directly explored by tests with scattering neutrons). The comparison of the spectra obtained both theoretically and experimentally makes it possible to conclude whether the model describes the real system efficiently. Comparing the spectra helps one to estimate the value of microscopic parameters.

Secondly, at a low temperature the primary contribution of the exchange spin interaction to thermodynamic characteristics of magnetic systems is given by longwave magnon excitations. The magnon excitations provide an essential contribution to electrical resistance of magnetic metals within a wide temperature region 
[2-7]. In the formation of electron-electron interactions with the use of magnetic subsystems the spin-wave excitations play an important role [8]. This is an unavoidable circumstance when the superconduction of fuses of rear-earth metals and transition elements are studied. We would like to note that an effective nucleus spin interaction is formed with collective electron excitations of electron shells [9]. Therefore, information about the type of magnon excitations is important for the purpose of a nuclear magnetic resonance.

This brief view of the phenomena and processes connected with magnons, shows great importance of magnon analyses. The calculation of spin excitations in the $s-d$ model is not new, but essential success was achieved just for two limited cases: narrow and wide conductive bands. We can assume the relation $x=|I| S / W$ to be the quantity criteria. Here $I$ is the parameter of the spin-electron exchange interaction, $S$ is the value of the localized atomic spin $(1 / 2 \leqslant S \leqslant \infty), W$ is the width of the conductive band.

The first investigations of spin waves in the $s-d$ model concerned the systems with a wide conduction band (magnetic metals) and parameter $x \ll 1$. If we describe the electron subsystem of metal within the free electron approximation, the relation $|I| S / E_{F}$ is a small parameter. Here $E_{F}$ is the Fermy energy. It is shown in [10] that it is possible to obtain an equation for the spin-wave spectrum by passing to the Bose representation of spin operators and splitting the equation of motion for double-time temperature Green functions. The spectrum of magnon excitations consists of two branches of optical and acoustic type. This result was confirmed later by using a diagram method for the Matsubara-Green function [11]. It is demonstrated in [12] that the form of spectra of a spin wave in degenerated semi-conductors is similar to the spin-wave spectrum of band ferromagnetic. This work explores two extreme cases: low density of current barriers and a half-completed conduction band. Elementary excitations in an anisotropic magnetic semiconductor were studied in [13]. The low density spectrum of Fermiand Bose-type excitations was calculated. The existence of two branches of excitations was demonstrated. The contribution of these branches to low temperature magnetization for different types of anisotropy was analyzed.

Papers [14-18] are dedicated to magnon excitations spectra for the narrowband magnetic semiconductors. In paper [14] the existence of an acoustic and optical branches was demonstrated by using an approximation similar to the random phases approximation. The hierarchy of equations for the calculation of magnetization and critical temperature was obtained in the approximation of the molecular field. An equation for the magnon Green function was obtained in the second approximation with respect to the parameter of $s-d$ exchange interaction. The results are presented in [16-18].

A number of tasks were considered in [19-21] by applying the Hartree-Fock approximation. Among them were magnetization, a spin-wave spectrum, magnonphonon interaction, the elementary magnetic excitations life-time and others. In this approximation we have found that magnetization achieves its maximum value when $W=0$ ( $W$ is the conduction band width). When the conduction band width 
increases, the magnetization and the critical temperature decrease.

The purpose of our work is to calculate the spin wave spectrum for crystaline $s-d$ models. The application of the spin Green functions makes it possible to obtain an equation for the spectrum in a long-wave region. The results obtained for crystal systems are the generalization of the research carried out in [15-16]. In particular, we investigated the influence of the Coulomb interaction between electrons on the spectrum and its damping.

\section{Hamiltonian of a generalized $s-d$ model}

Here we explore a model which describes the connection of localized atomic spins and the conductive electrons subsystem by exchange interaction. Atomic coordinates $\boldsymbol{R}_{1}, \ldots, \boldsymbol{R}_{N}$ may take on any value within the volume $V \subset \mathbb{R}^{3}$. At the same time it is assumed that space atomic distribution is homogenous and may be characterized by the atomic density $\rho=N / V$ in the thermodynamic limit.

The initial model Hamiltonian in the site representation is:

$$
\begin{aligned}
\hat{H}= & \sum_{1 \leqslant j \leqslant N} \sum_{\sigma} \mathcal{E}_{j, \sigma} \hat{n}_{j \sigma}-g \mu_{\mathrm{B}} h \sum_{1 \leqslant j \leqslant N} S_{j}^{z}+\sum_{1 \leqslant i \neq j \leqslant N} \sum_{\sigma} T_{i j} a_{i \sigma}^{+} a_{j \sigma}+ \\
& \left.+\frac{U}{2} \sum_{1 \leqslant j \leqslant N} \sum_{\sigma} \hat{n}_{j \sigma} \hat{n}_{j-\sigma}+\frac{I}{2} \sum_{1 \leqslant j \leqslant N} \sum_{\sigma}\left(\sigma S_{j}^{z} \hat{n}_{j \sigma}+S_{j}^{\sigma} a_{j,-\sigma}^{+} a_{j \sigma}\right)\right)- \\
& -\frac{1}{2} \sum_{1 \leqslant i \neq j \leqslant N} J_{i j}\left(S_{i}^{z} S_{j}^{z}+\frac{1}{2}\left(S_{i}^{+} S_{j}^{-}+S_{i}^{-} S_{j}^{+}\right)\right) .
\end{aligned}
$$

Here $a_{j \sigma}^{+}\left(a_{j \sigma}\right)$ are the creation and annihilation operators of the electrons in localized $s$-states of the atom situated in point $\boldsymbol{R}_{j}$ of the volume $V$. The index $\sigma$ takes values $\sigma=\uparrow, \downarrow$ (or $\sigma= \pm 1$ ) according to spin orientation to quantization axis $O Z$.

The coefficients that are diagonal with respect to site indexes

$$
\mathcal{E}_{j, \sigma}=\mathcal{E}_{j}-\frac{1}{2} \sigma \mu_{\mathrm{B}} h, \quad \sigma= \pm 1
$$

characterize the energy value of single-electron levels of the $j$-th atom when the external magnetic field $h$ is applied. $\mu_{\mathrm{B}}$ is the Bohr magneton.

The state of the electron localized on the $j$-th atom may be described by the $s$-type hydrogen wave function

$$
\psi_{j}(\boldsymbol{r})=\sqrt{\frac{1}{\pi a^{3}}} \exp \left(-\frac{\left|\boldsymbol{r}-\boldsymbol{R}_{j}\right|}{a}\right) .
$$

Here $a$ - is the effective Bohr radius. The integral

$$
S_{i j}=\int_{V} \psi_{i}^{*}(\boldsymbol{r}) \psi_{j}(\boldsymbol{r}) \mathrm{d} \boldsymbol{r}, \quad \boldsymbol{R}_{i} \neq \boldsymbol{R}_{j}
$$


is a quantitative characteristic of the overlap degree for orbits of different atoms. The penetration integral in the $0 \leqslant S_{i j} \leqslant 1$ interval depends on the wave function hybridization range. For orbital (3) the integral $S_{i j}$ may be expressed as

$$
S_{i j}=\left[1+\frac{\left|\boldsymbol{R}_{i}-\boldsymbol{R}_{j}\right|}{a}+\frac{1}{3} \frac{\left|\boldsymbol{R}_{i}-\boldsymbol{R}_{j}\right|^{2}}{a^{2}}\right] \exp \left(-\frac{\left|\boldsymbol{R}_{i}-\boldsymbol{R}_{j}\right|}{a}\right) .
$$

The narrow conduction bands yield small overlap integrals values. We can assume the relation $a / \bar{R}_{0}=\left(4 / 3 \pi \rho a^{3}\right)^{1 / 3}$ to be quantitative criteria of the wave functions overlap. Here $\bar{R}_{0}=(3 / 4 \pi \rho)^{1 / 3}$ means an average distance between the atoms if their distribution in the volume $V$ is homogenous, $\rho$ is the atomic density.

Matrix elements of the transition between states presented by wave functions (3) are of the following analytical form:

$$
T_{i j}=T_{0}\left[1+\frac{\left|\boldsymbol{R}_{i}-\boldsymbol{R}_{j}\right|}{a}\right] \exp \left(-\frac{\left|\boldsymbol{R}_{i}-\boldsymbol{R}_{j}\right|}{a}\right),
$$

here $T_{0}$ - is the energy of the atomic level.

Model (1) may describe the electron and magnetic properties of numerous objects like crystals, displacement alloys, amorphous compositions, magnetic metals alloys. The atomic levels $\varepsilon_{j}$ may take on random values (a diagonal disorder) for structurally disordered models. Matrix elements $T_{i j} \equiv T\left(\left|\boldsymbol{R}_{i}-\boldsymbol{R} j\right|\right)$, which are integrals of electron skipping between atoms $\boldsymbol{R}_{i}$ and $\boldsymbol{R}_{j}$, take random values, too (a non-diagonal disorder).

The intensity of spin-spin exchange interaction is described by the parameter $J_{i j} \equiv J\left(\mid \boldsymbol{R}_{i}-\boldsymbol{R}_{j}\right)$. It is assumed that $J_{i j}$ is positive for any inter-atomic distances if $\left|\boldsymbol{R}_{i}-\boldsymbol{R}_{j}\right| \geqslant 0$. The parameter $I$ of the spin-electron interaction may be either positive or negative. The Coulomb integral $U$ describes the intensity of repulsion between electrons of the opposite spin localized on the same $s$ - orbital. The electron interaction is assumed to be short-range, similarly to the Stoner model.

\section{Equation of motion for the Green's function of the $s-d$ model}

Let us determine the electron spin operators by the relations:

$$
\begin{gathered}
m_{j}^{z}=\frac{1}{2} \sum_{\sigma= \pm 1} \sigma \hat{n}_{j \sigma} \equiv \frac{1}{2}\left(\hat{n}_{j \uparrow}-n_{j \downarrow}\right), \\
m_{j}^{+}=a_{j \uparrow}^{+} a_{j \downarrow}, \quad m_{j}^{-}=a_{j \downarrow}^{+} a_{j \uparrow} .
\end{gathered}
$$

The operator $m_{j}^{z}$ means the spin polarization operator of the atom situated in point $\boldsymbol{R}_{j}$. In their turn, operators $m_{j}^{ \pm}$describe the process of reorientation to the quantum axis. The commutation relations for operators (7) are the following:

$$
\left[m_{i}^{z}, m_{j}^{\sigma}\right]=\sigma m_{i}^{\sigma} \delta_{i j}, \quad\left[m_{i}^{\sigma}, m_{j}^{\sigma^{\prime}}\right]=2 \sigma m_{i}^{z} \delta_{i j} \delta_{\sigma,-\sigma^{\prime}}, \quad \sigma, \sigma^{\prime}= \pm 1
$$


The algebra for operators $m_{j}^{z}, m_{j}^{ \pm}$is similar to the algebra for operators $S_{j}^{z}, S_{j}^{ \pm}$ of localized atomic spins.

It is necessary to calculate the matrix of double-time temperature Green functions in a frequency representation to be able to describe spin excitations in model (1)

$$
\hat{\mathbb{K}}_{i j}(\omega) \equiv\left[\begin{array}{ll}
\left\langle\left\langle S_{i}^{+} \mid S_{j}^{-}\right\rangle\right\rangle_{\omega} & \left\langle\left\langle m_{i}^{+} \mid S_{j}^{-}\right\rangle\right\rangle_{\omega} \\
\left\langle\left\langle S_{i}^{+} \mid m_{j}^{-}\right\rangle\right\rangle_{\omega} & \left\langle\left\langle m_{i}^{+} \mid m_{j}^{-}\right\rangle\right\rangle_{\omega}
\end{array}\right]
$$

The components of matrix $\hat{\mathbb{K}}_{i j}(\omega)$ are related to double-time retarded Green functions by the Fourier transformation:

$$
\hat{\mathbb{K}}_{i j}(\omega)=\frac{1}{2 \pi} \int_{-\infty}^{\infty} \mathrm{d}\left(t-t^{\prime}\right) \mathbb{K}_{i j}\left(t-t^{\prime}\right) \exp \left[i \omega\left(t-t^{\prime}\right)\right] .
$$

The double-time retarted Green function is by definition:

$$
\hat{\mathbb{K}}(t-t) \equiv\left\langle\left\langle\hat{A}_{i}(t) \mid \hat{B}_{j}\left(t^{\prime}\right)\right\rangle\right\rangle_{\omega}=-i \Theta\left(t-t^{\prime}\right)\left\langle\left[\hat{A}_{i}(t), \hat{B}_{j}\left(t^{\prime}\right)\right]_{-}\right\rangle .
$$

Here $\hat{A}_{i}(t)$ i $\hat{B}_{j}\left(t^{\prime}\right)$ denote the corresponding operators from a set of spin operators on which function (9) is defined. The operators depend on time via the Heisenberg representation. Symbol $\langle(\ldots)\rangle$ means thermodynamic averaging over the Gibbs distribution with the Hamiltonian (6).

The Fourier components (10) of Green functions (11) correspond to the following equations of motion:

$$
\omega\left\langle\left\langle\hat{A}_{i} \mid \hat{B}_{j}\right\rangle\right\rangle_{\omega}=\frac{1}{2 \pi}\left\langle\left[\hat{A}_{i}, \hat{B}_{j}\right]_{-}\right\rangle+\left\langle\left\langle\left[\hat{A}_{i}, \hat{H}\right]_{-} \mid \hat{B}_{j}\right\rangle\right\rangle_{\omega} .
$$

The correlator $\left\langle\hat{B}_{j}\left(t^{\prime}\right) \hat{A}_{i}(t)\right\rangle$ is obtained from the spectral theorem

$$
\left\langle\hat{B}_{j}\left(t^{\prime}\right) \hat{A}_{i}(t)\right\rangle=i \lim _{\epsilon \rightarrow 0} \int_{-\infty}^{\infty} \frac{\left\langle\left\langle\hat{A}_{i} \mid \hat{B}_{j}\right\rangle\right\rangle_{\omega+i \varepsilon}-\left\langle\left\langle\hat{A}_{i} \mid \hat{B}_{j}\right\rangle\right\rangle_{\omega-i \varepsilon}}{e^{\beta \omega}-1} e^{-i \omega\left(t-t^{\prime}\right)} \mathrm{d} \omega
$$

where $\beta=\left(k_{\mathrm{B}} T\right)^{-1}$ - is the reciprocal temperature in energy units.

For the spin Green function (9) we obtain the following system of equations of motion :

$$
\begin{gathered}
\left(\omega-g \mu_{\mathrm{B}} h+I m_{i}\right)\left\langle\left\langle S_{i}^{+} \mid S_{j}^{-}\right\rangle\right\rangle_{\omega}=\frac{1}{2 \pi} 2 M_{i} \delta_{i j}+I M_{i}\left\langle\left\langle m_{i}^{+} \mid S_{j}^{-}\right\rangle\right\rangle_{\omega}+ \\
+\sum_{1 \leqslant n \leqslant N} J_{i n}\left(M_{n}\left\langle\left\langle S_{i}^{+} \mid S_{j}^{-}\right\rangle\right\rangle_{\omega}-M_{i}\left\langle\left\langle S_{n}^{+} \mid S_{j}^{-}\right\rangle\right\rangle_{\omega}\right), \\
\left(\omega-\mu_{\mathrm{B}} h+I M_{i}\right)\left\langle\left\langle m_{i}^{+} \mid m_{j}^{-}\right\rangle\right\rangle_{\omega}=\frac{1}{2 \pi} 2 m_{i} \delta_{i j}+I m_{i}\left\langle\left\langle S_{i}^{+} \mid m_{j}^{-}\right\rangle\right\rangle_{\omega}+ \\
+\sum_{1 \leqslant n \leqslant N} T_{i n}\left(Q_{i n, j}^{\uparrow}(\omega)-Q_{n i, j}^{\uparrow}(\omega)\right)
\end{gathered}
$$




$$
\begin{gathered}
\left(\omega-g \mu_{\mathrm{B}} h+I m_{i}\right)\left\langle\left\langle S_{i}^{+} \mid m_{j}^{-}\right\rangle\right\rangle_{\omega}=I M_{i}\left\langle\left\langle m_{i}^{+} \mid m_{j}^{-}\right\rangle\right\rangle_{\omega}+ \\
+\sum_{1 \leqslant n \leqslant N} J_{i n}\left(M_{n}\left\langle\left\langle S_{i}^{+} \mid m_{j}^{-}\right\rangle\right\rangle_{\omega}-M_{i}\left\langle\left\langle S_{n}^{+} \mid m_{j}^{-}\right\rangle\right\rangle_{\omega}\right), \\
\left(\omega-\mu_{\mathrm{B}} h+I M_{i}\right)\left\langle\left\langle m_{i}^{+} \mid S_{j}^{-}\right\rangle\right\rangle_{\omega}=I m_{i}\left\langle\left\langle S_{i}^{+} \mid S_{j}^{-}\right\rangle\right\rangle_{\omega}+ \\
+\sum_{1 \leqslant n \leqslant N} T_{i n}\left(P_{i n, j}^{\uparrow}(\omega)-P_{n i, j}^{\uparrow}(\omega)\right) .
\end{gathered}
$$

In this equation we use the following notation $M_{i}=\left\langle S_{i}^{z}\right\rangle, m_{i}=\left\langle m_{i}^{z}\right\rangle$. The quantities $M_{i}$ and $m_{i}$ are the average thermodynamic values of magnetization of a localized spin and the electron-spin polarization of an atom at $\boldsymbol{R}_{j} \in V$ respectively. We designate the three-center spin-electron Green functions by $P_{n i, j}^{\sigma}(\omega)$ and $Q_{n i, j}^{\sigma}(\omega)$ :

$$
\begin{array}{cc}
P_{i n, j}^{\sigma}(\omega)=\left\langle\left\langle a_{i \sigma}^{+} a_{n,-\sigma} \mid S_{j}^{-}\right\rangle\right\rangle_{\omega}, & \sigma=\uparrow, \downarrow, \\
Q_{i n, j}^{\sigma}(\omega)=\left\langle\left\langle a_{i \sigma}^{+} a_{n,-\sigma} \mid m_{j}^{-}\right\rangle\right\rangle_{\omega}, & \sigma=\uparrow, \downarrow .
\end{array}
$$

The following approximations for higher-order Green functions are used to close the equation hierarchy:

$$
\begin{aligned}
&\left\langle\left\langle m_{i}^{z} S_{i}^{+} \mid \hat{B}_{j}\right\rangle\right\rangle_{\omega} \simeq m_{i}\left\langle\left\langle S_{i}^{+} \mid \hat{B}_{j}\right\rangle\right\rangle_{\omega}, \\
&\left\langle\left\langle S_{n}^{z} S_{i}^{+} \mid \hat{B}_{j}\right\rangle\right\rangle_{\omega} \simeq M_{n}\left\langle\left\langle S_{i}^{+} \mid \hat{B}_{j}\right\rangle\right\rangle_{\omega}, \\
&\left\langle\left\langle S_{i}^{z} m_{i}^{+} \mid \hat{B}_{j}\right\rangle\right\rangle_{\omega} \simeq M_{i}\left\langle\left\langle m_{i}^{+} \mid \hat{B}_{j}\right\rangle\right\rangle_{\omega}, \\
&\left\langle\left\langle S_{i}^{z} S_{n}^{+} \mid \hat{B}_{j}\right\rangle\right\rangle_{\omega} \simeq M_{i}\left\langle\left\langle S_{n}^{+} \mid \hat{B}_{j}\right\rangle\right\rangle_{\omega} .
\end{aligned}
$$

Writing $\hat{B}_{j}$ we mean the operator $S_{j}^{-}$or $m_{j}^{-}$. The approximation of type $(20)$ is known as the Tyablikov decoupling in the Heisenberg magnetic theory. The characteristic feature of this approximation is that factorization of higher-order Green functions is done for non-coinciding site indices $\left(\boldsymbol{R}_{i} \neq \boldsymbol{R}_{n}\right)$ of spin operators. The Tyablikov approximation yields wrong results for coinciding site indices. The physical meaning and the region of validity of this approximation are thoroughly discussed in [5]. We note that in relations (20) approximation is made for similar operator site indices. But these operators belong to different subsystems and commute with each other, so this approximation is equivalent to the Tyablikov decoupling procedure.

The equation for the three-center Green functions (18) is

$$
\begin{aligned}
(\omega- & \left.Z_{i n}^{\sigma}\right) P_{i n, j}^{\sigma}(\omega)=\sum_{1 \leqslant l \leqslant N}\left(T_{n l} P_{i l, j}^{\sigma}(\omega)-T_{l i} P_{l n, j}^{\sigma}(\omega)\right)+ \\
& +\frac{1}{2} I \delta_{\sigma, \uparrow}\left(f_{i n}^{\uparrow}\left\langle\left\langle S_{n}^{+} \mid S_{j}^{-}\right\rangle\right\rangle_{\omega}-f_{i n}^{\downarrow}\left\langle\left\langle S_{i}^{+} \mid S_{j}^{-}\right\rangle\right\rangle_{\omega}\right)+ \\
& +\frac{1}{2} I \delta_{\sigma, \downarrow}\left(f_{i n}^{\downarrow}\left\langle\left\langle S_{n}^{+} \mid S_{j}^{-}\right\rangle\right\rangle_{\omega}-f_{i n}^{\uparrow}\left\langle\left\langle S_{i}^{+} \mid S_{j}^{-}\right\rangle\right\rangle_{\omega}\right)- \\
& -m U \delta_{i n}\left\langle\left\langle a_{i \sigma}^{+} a_{i,-\sigma} \mid S_{j}^{-}\right\rangle\right\rangle_{\omega},
\end{aligned}
$$


and the equation for function (19) is

$$
\begin{aligned}
(\omega- & \left.Z_{i n}^{\sigma}\right) Q_{i n, j}^{\sigma}(\omega)=\frac{1}{2 \pi}\left(\delta_{n j} f_{i n}^{\sigma}-\delta_{i j} f_{i n}^{-\sigma}\right)+ \\
& +\sum_{1 \leqslant l \leqslant N}\left(T_{n l} Q_{i l, j}^{\sigma}(\omega)-T_{l i} Q_{l n, j}^{\sigma}(\omega)\right)+ \\
& +\frac{1}{2} I \delta_{\sigma, \uparrow}\left(f_{i n}^{\uparrow}\left\langle\left\langle S_{n}^{+} \mid m_{j}^{-}\right\rangle\right\rangle_{\omega}-f_{i n}^{\downarrow}\left\langle\left\langle S_{i}^{+} \mid m_{j}^{-}\right\rangle\right\rangle_{\omega}\right)+ \\
& +\frac{1}{2} I \delta_{\sigma, \downarrow}\left(f_{i n}^{\downarrow}\left\langle\left\langle S_{n}^{+} \mid m_{j}^{-}\right\rangle\right\rangle_{\omega}-f_{i n}^{\uparrow}\left\langle\left\langle S_{i}^{+} \mid m_{j}^{-}\right\rangle\right\rangle_{\omega}\right)- \\
& -m U \delta_{i n}\left\langle\left\langle a_{i \sigma}^{+} a_{i,-\sigma} \mid m_{j}^{-}\right\rangle\right\rangle_{\omega} .
\end{aligned}
$$

Here

$$
Z_{i n}^{\sigma}=\mathcal{E}_{n}-\mathcal{E}_{i}+\sigma \mu_{\mathrm{B}} h+U\left(1-\delta_{i n}\right)\left[\left\langle\hat{n}_{n \sigma}\right\rangle-\left\langle\hat{n}_{i,-\sigma}\right\rangle\right]-\frac{1}{2} I \sigma\left(M_{n}+M_{i}\right),
$$

and the correlation function

$$
f_{i n}^{\sigma}=\left\langle a_{i \sigma}^{+} a_{n \sigma}\right\rangle, \quad \sigma=\uparrow, \downarrow .
$$

We apply the Hartree-Fock approximation and the decoupling for higher-order Green functions similar to (20) to close the equation hierarchy (21)-(22).

On the basis of the obtained equation one immediately gets an equation for the Green functions $\left\langle\left\langle S_{i}^{+} \mid S_{j}^{-}\right\rangle\right\rangle_{\omega}$ and $\left\langle\left\langle m_{i}^{+} \mid m_{j}^{-}\right\rangle\right\rangle_{\omega}$, that yield a spin excitation spectrum. After some algebraic transformation one obtains the following equation for function $\left\langle\left\langle S_{i}^{+} \mid S_{j}^{-}\right\rangle\right\rangle_{\omega}$ :

$$
\begin{array}{r}
\left(\omega-g \mu_{\mathrm{B}} h+I_{i} \frac{\omega-\mu_{\mathrm{B}} h}{\omega-\mu_{\mathrm{B}} h+I M_{i}}\right)\left\langle\left\langle S_{i}^{+} \mid S_{j}^{-}\right\rangle\right\rangle_{\omega}=2 M_{i} \frac{\delta_{i j}}{2 \pi}+ \\
+\frac{I M_{i}}{\omega-\mu_{\mathrm{B}} h+I M_{i}} \sum_{1 \leqslant n \leqslant N} T_{i n}\left[P_{i n, j}^{\uparrow}(\omega)-P_{n i, j}^{\uparrow}(\omega)\right]+ \\
\quad+\sum_{1 \leqslant n \leqslant N} J_{i n}\left[M_{n}\left\langle\left\langle S_{i}^{+} \mid S_{j}^{-}\right\rangle\right\rangle_{\omega}-M_{i}\left\langle\left\langle S_{n}^{+} \mid S_{j}^{-}\right\rangle\right\rangle_{\omega}\right],
\end{array}
$$

and for function $\left\langle\left\langle m_{i}^{+} \mid m_{j}^{-}\right\rangle\right\rangle_{\omega}$

$$
\begin{array}{r}
\left(\omega-\mu_{\mathrm{B}} h+I M_{i} \frac{\omega-g \mu_{\mathrm{B}} h}{\omega-g \mu_{\mathrm{B}} h+I m_{i}}\right)\left\langle\left\langle m_{i}^{+} \mid m_{j}^{-}\right\rangle\right\rangle_{\omega}=2 m_{i} \frac{\delta_{i j}}{2 \pi}+ \\
+\frac{I^{2} m_{i}}{\omega-g \mu_{\mathrm{B}} h+I m_{i}} \sum_{1 \leqslant n \leqslant N} J_{i n}\left[\frac{M_{i} M_{n}}{\omega-g \mu_{\mathrm{B}} h+I m_{i}}\left\langle\left\langle m_{i}^{+} \mid m_{j}^{-}\right\rangle\right\rangle_{\omega}-\right. \\
\left.-\frac{M_{i} M_{n}}{\omega-g \mu_{\mathrm{B}} h+I m_{n}}\left\langle\left\langle m_{n}^{+} \mid m_{j}^{-}\right\rangle\right\rangle_{\omega}\right]+\sum_{1 \leqslant n \leqslant N} T_{i n}\left[Q_{i n, j}^{\uparrow}(\omega)-Q_{n i, j}^{\uparrow}(\omega)\right]
\end{array}
$$

respectively. The calculation of the Green functions with the help of the obtained equations demands the definition of the structure disorder. 
It is apparent that the written equations are not sufficient for a self-consistent calculation of the spin-wave spectrum. Particularly, we need to calculate the oneelectron Green function

$$
G_{i j, \sigma}(\omega)=\left\langle\left\langle a_{i \sigma} \mid a_{j \sigma}^{+}\right\rangle\right\rangle_{\omega}=-i \Theta\left(t-t^{\prime}\right)\left\langle\left[a_{i \sigma}(t), a_{j \sigma}\left(t^{\prime}\right)\right]_{+}\right\rangle .
$$

to calculate correlators $f_{i, n}^{\sigma}$ and $\left\langle\hat{n}_{i \sigma}\right\rangle$. If the decoupling similar to the mentioned one is applied, the equation for the one-electron Green function may be written in the next form:

$$
\left(\omega-\Pi_{i \sigma}\right) G_{i j, \sigma}(\omega)=\frac{1}{2 \pi} \delta_{i j}+\sum_{1 \leqslant n \leqslant N} T_{i n} G_{i n, \sigma}(\omega),
$$

Here the value

$$
\Pi_{i \sigma}=\mathcal{E}_{i}-\frac{\sigma \mu_{\mathrm{B}} h}{2}+U\left\langle\hat{n}_{i,-\sigma}\right\rangle+\frac{1}{2} \sigma I M_{i}
$$

characterizes the energy of the electron localized on the $i$-th atom. Now equations (14)-(17), (21)-(22) and (27) form a closed hierarchy.

\section{Equations for the magnon excitations spectrum}

For the crystalline structure of the initial $s-d$ model one has $M_{i}=M, m_{i}=$ $m$, and $\left\langle\hat{n}_{i \sigma}\right\rangle=n_{\sigma}$ for all sites of the lattice. These relations hold due to the translational symmetry of the crystal structure.

It is more convenient to work with Green functions in the momentum space, making decomposition of the spin and Fermi operators to Fourier series on the lattice:

$$
\begin{aligned}
& S_{j}^{ \pm}=\frac{1}{\sqrt{N}} \sum_{\boldsymbol{k}} S_{\boldsymbol{k}}^{ \pm} e^{ \pm i \boldsymbol{k} \boldsymbol{R}_{j}}, \\
& a_{j \sigma}^{ \pm}=\frac{1}{\sqrt{N}} \sum_{\boldsymbol{k}} a_{\boldsymbol{k} \sigma}^{ \pm} e^{\mp i \boldsymbol{k} \boldsymbol{R}_{j}}
\end{aligned}
$$

Here operator $a_{j \sigma}^{-} \equiv a_{j \sigma}$. The wave vector $\boldsymbol{k}$ takes its values within the first Brillouin zone. The inverse to (29) Fourier transformation reads:

$$
\begin{aligned}
S_{\boldsymbol{k}}^{ \pm} & =\frac{1}{\sqrt{N}} \sum_{1 \leqslant j \leqslant N} S_{j}^{ \pm} e^{\mp i \boldsymbol{k} \boldsymbol{R}_{j}}, \\
a_{\boldsymbol{k} \sigma}^{ \pm} & =\frac{1}{\sqrt{N}} \sum_{1 \leqslant j \leqslant N} a_{j \sigma}^{ \pm} e^{ \pm i \boldsymbol{k} \boldsymbol{R}_{j}} .
\end{aligned}
$$

Then, equation (25) in the momentum representation takes the form:

$$
\left(\omega-g \mu_{\mathrm{B}} h+\frac{\operatorname{Im}\left(\omega-\mu_{\mathrm{B}} h\right)}{\omega-\mu_{\mathrm{B}} h+I M}-M\left(J_{0}-J_{k}\right)\right)\left\langle\left\langle S_{\boldsymbol{k}}^{+} \mid S_{\boldsymbol{q}}^{-}\right\rangle\right\rangle_{\omega}=\frac{\delta_{\boldsymbol{k}, \boldsymbol{q}}}{2 \pi} 2 M+
$$




$$
+\frac{I M}{\omega-\mu_{\mathrm{B}} h+I M} \frac{1}{\sqrt{N}} \sum_{\boldsymbol{p}} t_{p}\left(\left\langle\left\langle a_{\boldsymbol{p}-\boldsymbol{k}, \uparrow}^{+} a_{\boldsymbol{p}, \downarrow} \mid S_{\boldsymbol{q}}^{-}\right\rangle\right\rangle_{\omega}-\left\langle\left\langle a_{\boldsymbol{p}, \uparrow}^{+} a_{\boldsymbol{p}+\boldsymbol{k}, \downarrow} \mid S_{\boldsymbol{q}}^{-}\right\rangle\right\rangle_{\omega}\right) .
$$

and equation (26) can be written as

$$
\begin{gathered}
\left(\omega-\mu_{\mathrm{B}} h+\frac{I M\left(\omega-g \mu_{\mathrm{B}} h\right)}{\omega-g \mu_{\mathrm{B}} h+I m}-\frac{I^{2} M^{2} m\left(J_{0}-J_{k}\right)}{\left(\omega-g \mu_{\mathrm{B}} h+I m\right)^{2}}\right)\left\langle\left\langle m_{\boldsymbol{k}}^{+} \mid m_{\boldsymbol{q}}^{-}\right\rangle\right\rangle_{\omega}=\frac{\delta_{\boldsymbol{k}, \boldsymbol{q}}}{2 \pi} 2 m+ \\
+\frac{1}{\sqrt{N}} \sum_{\boldsymbol{p}} t_{p}\left(\left\langle\left\langle a_{\boldsymbol{p}-\boldsymbol{k}, \uparrow}^{+} a_{\boldsymbol{p}, \downarrow} \mid m_{\boldsymbol{q}}^{-}\right\rangle\right\rangle_{\omega}-\left\langle\left\langle a_{\boldsymbol{p}, \uparrow}^{+} a_{\boldsymbol{p}+\boldsymbol{k}, \downarrow} \mid m_{\boldsymbol{q}}^{-}\right\rangle\right\rangle_{\omega}\right) .
\end{gathered}
$$

Here the Fourier coefficients of exchange interaction and the matrix element of the hopping integral have the form:

$$
\begin{aligned}
J_{k} & =\frac{1}{N} \sum_{1 \leqslant i, j \leqslant N} J_{i j} e^{-i \boldsymbol{k}\left(\boldsymbol{R}_{i}-\boldsymbol{R}_{j}\right)}, \\
t_{k} & =\frac{1}{N} \sum_{1 \leqslant i, j \leqslant N} T_{i j} e^{-i \boldsymbol{k}\left(\boldsymbol{R}_{i}-\boldsymbol{R}_{j}\right)} .
\end{aligned}
$$

The inverse to relation (33) Fourier transformation reads:

$$
\begin{aligned}
& J_{i n}=\frac{1}{N} \sum_{\boldsymbol{k}} J_{k} e^{i \boldsymbol{k}\left(\boldsymbol{R}_{i}-\boldsymbol{R}_{n}\right)}, \\
& T_{\text {in }}=\frac{1}{N} \sum_{\boldsymbol{k}} t_{k} e^{i \boldsymbol{k}\left(\boldsymbol{R}_{i}-\boldsymbol{R}_{n}\right)} .
\end{aligned}
$$

\subsection{Calculation of the function $\left\langle\left\langle S_{k}^{+} \mid S_{q}^{-}\right\rangle\right\rangle_{\omega}$}

The diagonal matrix elements of the Green function $\left\langle\left\langle S_{\boldsymbol{k}}^{+} \mid S_{\boldsymbol{q}}^{-}\right\rangle\right\rangle_{\omega}$ are also connected with site functions $\left\langle\left\langle S_{i}^{+} \mid S_{j}^{-}\right\rangle\right\rangle_{\omega}$ via the Fourier transformation

$$
\begin{gathered}
\left\langle\left\langle S_{i}^{+} \mid S_{j}^{-}\right\rangle\right\rangle_{\omega}=\frac{1}{N} \sum_{\boldsymbol{k}}\left\langle\left\langle S_{\boldsymbol{k}}^{+} \mid S_{\boldsymbol{k}}^{-}\right\rangle\right\rangle_{\omega} e^{\imath \boldsymbol{k}\left(\boldsymbol{R}_{i}-\boldsymbol{R}_{j}\right)}, \\
\left\langle\left\langle S_{\boldsymbol{k}}^{+} \mid S_{\boldsymbol{k}}^{-}\right\rangle\right\rangle_{\omega}=\frac{1}{N} \sum_{1 \leq i, j \leq N}\left\langle\left\langle S_{i}^{+} \mid S_{j}^{-}\right\rangle\right\rangle_{\omega} e^{-\imath \boldsymbol{k}\left(\boldsymbol{R}_{i}-\boldsymbol{R}_{j}\right)} .
\end{gathered}
$$

The right-hand side of equation (31) includes the function $\left\langle\left\langle a_{\boldsymbol{k}, \uparrow}^{+} a_{\boldsymbol{p}, \downarrow} \mid S_{\boldsymbol{q}}^{-}\right\rangle\right\rangle_{\omega}$, that is a Fourier transform of the Green function $P_{i n, j}^{\sigma}(\omega)$. For this function, on the basis of (21), one easily obtains the equation:

$\left\langle\left\langle a_{\boldsymbol{k}, \uparrow}^{+} a_{\boldsymbol{p}, \downarrow} \mid S_{\boldsymbol{q}}^{-}\right\rangle\right\rangle_{\omega}=\frac{I}{2 \sqrt{N}} \frac{f_{k}^{\uparrow}-f_{p}^{\downarrow}}{\Lambda(\omega)-t_{p}+t_{k}}\left\langle\left\langle S_{\boldsymbol{p}-\boldsymbol{k}}^{+} \mid S_{\boldsymbol{q}}^{-}\right\rangle\right\rangle_{\omega}-\frac{2 U m}{\Lambda(\omega)-t_{p}-t_{k}} M^{\uparrow}(\boldsymbol{k}, \boldsymbol{p} ; \boldsymbol{q} \mid \omega)$. 
Here

$$
f_{k}^{\sigma}=\frac{1}{N} \sum_{1 \leqslant i, j \leqslant N} f_{i j}^{\sigma} e^{-i \boldsymbol{k}\left(\boldsymbol{R}_{i}-\boldsymbol{R}_{j}\right)}
$$

is the distribution function of electrons in the momentum space.

The last term in the right-hand side of equation (36) contains the function

$$
M^{\uparrow}(\boldsymbol{k}, \boldsymbol{p} ; \boldsymbol{q} \mid \omega) \equiv M^{\uparrow}(\boldsymbol{p}-\boldsymbol{k} ; \boldsymbol{q} \mid \omega)=\frac{1}{N} \sum_{\boldsymbol{g}}\left\langle\left\langle a_{\boldsymbol{g}+\boldsymbol{k}, \uparrow}^{+} a_{\boldsymbol{g}+\boldsymbol{p}, \downarrow} \mid S_{\boldsymbol{q}}^{-}\right\rangle\right\rangle_{\omega},
$$

that satisfies the following integral equation:

$$
\begin{gathered}
M^{\uparrow}(\boldsymbol{k}, \boldsymbol{p} ; \boldsymbol{q} \mid \omega)=\frac{1}{2 \sqrt{N}} A(\boldsymbol{k}, \boldsymbol{p} \mid \omega)\left\langle\left\langle S_{\boldsymbol{p}-\boldsymbol{k}}^{+} \mid S_{\boldsymbol{q}}^{-}\right\rangle\right\rangle_{\omega}- \\
-\frac{2 U m}{N} \sum_{\boldsymbol{g}} \frac{1}{\Lambda(\omega)-t_{\boldsymbol{g}+\boldsymbol{p}}+t_{\boldsymbol{g}+\boldsymbol{k}}} M^{\uparrow}(\boldsymbol{g}+\boldsymbol{k}, \boldsymbol{g}+\boldsymbol{p} ; \boldsymbol{q} \mid \omega) .
\end{gathered}
$$

Here coefficients

$$
A(\boldsymbol{k}, \boldsymbol{p} \mid \omega) \equiv A(\boldsymbol{p}-\boldsymbol{k} \mid \omega)=\frac{1}{N} \sum_{\boldsymbol{g}} \frac{f_{\boldsymbol{g}+\boldsymbol{k}}^{\uparrow}-f_{\boldsymbol{g}+\boldsymbol{p}}^{\downarrow}}{\Lambda(\omega)-t_{\boldsymbol{g}+\boldsymbol{p}}+t_{\boldsymbol{g}+\boldsymbol{k}}}
$$

and $\Lambda(\omega)=\omega-\mu_{\mathrm{B}} h+I M-2 U m$.

Thus, equations (31, (36) and (39) form a closed set of equations. From these equations one can find the spin Green function $\left\langle\left\langle S_{\boldsymbol{k}}^{+} \mid S_{\boldsymbol{q}}^{-}\right\rangle\right\rangle_{\omega}$. Let us make the simplest approximation in equation (39) by neglecting the integral term. In this approximation

$$
M^{\uparrow}(\boldsymbol{p}-\boldsymbol{k} ; \boldsymbol{q} \mid \omega) \simeq \frac{1}{2 \sqrt{N}} A(\boldsymbol{p}-\boldsymbol{k} \mid \omega)\left\langle\left\langle S_{\boldsymbol{p}-\boldsymbol{k}}^{+} \mid S_{\boldsymbol{q}}^{-}\right\rangle\right\rangle_{\omega}
$$

Neglecting the integral term in (39) is justified if $U / W \ll 1$, where $W$ - is the width of Stoner subbands. Such criteria agree with the condition of validity of the Hartree-Fock approximation. After some algebraic transformation one obtains the final result for the Green function:

$$
\left\langle\left\langle S_{\boldsymbol{k}}^{+} \mid S_{\boldsymbol{q}}^{-}\right\rangle\right\rangle_{\omega}=\frac{\omega-\mu_{\mathrm{B}} h+I M}{R(\boldsymbol{k} ; \omega)} \frac{M}{\pi} \delta_{\boldsymbol{k}, \boldsymbol{q}}
$$

Here the denominator is defined as

$$
\begin{gathered}
R(\boldsymbol{k}, \omega)=\left[\omega-g \mu_{\mathrm{B}} h-M\left(J_{0}-J_{k}\right)\right]\left[\omega-\mu_{\mathrm{B}} h+I M\right]+\operatorname{Im}\left(\omega-\mu_{\mathrm{B}} h\right)- \\
-\frac{I^{2} M}{2 N} \sum_{\boldsymbol{p}} \frac{\left(t_{\boldsymbol{p}+\boldsymbol{k}}-t_{\boldsymbol{p}}\right)\left(f_{\boldsymbol{p}}^{\uparrow}-f_{\boldsymbol{p}+\boldsymbol{k}}^{\downarrow}\right)}{\omega-E_{\boldsymbol{p}+\boldsymbol{k}, \downarrow}^{0}+E_{\boldsymbol{p}, \uparrow}^{0}}+ \\
+I^{2} M U m \chi_{0}^{+-}(\boldsymbol{k} \mid \omega) \frac{1}{N} \sum_{\boldsymbol{p}} \frac{t_{\boldsymbol{p}+\boldsymbol{k}}-t_{\boldsymbol{p}}}{\omega-E_{\boldsymbol{p}+\boldsymbol{k}, \downarrow}^{0}+E_{\boldsymbol{p}, \uparrow}^{0}}
\end{gathered}
$$


In equation (43) the function

$$
\chi_{0}^{+-}(\boldsymbol{k} \mid \omega)=\frac{1}{N} \sum_{\boldsymbol{p}} \frac{f_{\boldsymbol{p}}^{\uparrow}-f_{\boldsymbol{p}+\boldsymbol{k}}^{\downarrow}}{\omega-E_{\boldsymbol{p}+\boldsymbol{k}, \downarrow}^{0}+E_{\boldsymbol{p}, \uparrow}^{0}}
$$

is well known from the electron gas theory. In its terms, in particular, one can express the generalized transverse susceptibility of electron subsystems in metals [1-4]. The spectrum $E_{\boldsymbol{p}, \sigma}^{0}$ of band electrons is given below.

\subsection{Calculation of the function $\left\langle\left\langle m_{k}^{+} \mid m_{q}^{-}\right\rangle\right\rangle_{\omega}$}

For the function $\left\langle\left\langle a_{\boldsymbol{k}, \uparrow}^{+} a_{\boldsymbol{p}, \downarrow} \mid m_{\boldsymbol{q}}^{-}\right\rangle\right\rangle_{\omega}$, which is included in equation (32), we get the equation

$$
\begin{aligned}
& {\left[\Lambda(\omega)-t_{p}+t_{k}\right]\left\langle\left\langle a_{\boldsymbol{k}, \uparrow}^{+} a_{\boldsymbol{p}, \downarrow} \mid m_{\boldsymbol{q}}^{-}\right\rangle\right\rangle_{\omega}=\frac{\delta_{\boldsymbol{p}-\boldsymbol{k}, \boldsymbol{q}}}{2 \pi \sqrt{N}}\left(f_{k}^{\uparrow}-f_{p}^{\downarrow}\right)+} \\
& +\frac{I}{2 \sqrt{N}}\left(f_{k}^{\uparrow}-f_{p}^{\downarrow}\right)\left\langle\left\langle S_{\boldsymbol{p}-\boldsymbol{k}}^{+} \mid m_{\boldsymbol{q}}^{-}\right\rangle\right\rangle_{\omega}-2 U m N^{\uparrow}(\boldsymbol{k}, \boldsymbol{p} ; \boldsymbol{q} \mid \omega) .
\end{aligned}
$$

Here the Green function

$$
N^{\uparrow}(\boldsymbol{k}, \boldsymbol{p} ; \boldsymbol{q} \mid \omega) \equiv N^{\uparrow}(\boldsymbol{p}-\boldsymbol{k} ; \boldsymbol{q} \mid \omega)=\frac{1}{N} \sum_{\boldsymbol{g}}\left\langle\left\langle a_{\boldsymbol{g}+\boldsymbol{k}, \uparrow}^{+} a_{\boldsymbol{g}+\boldsymbol{p}, \downarrow} \mid m_{\boldsymbol{q}}^{-}\right\rangle\right\rangle_{\omega} .
$$

Let us use an approximation similar to the one used when we calculated functions $\left\langle\left\langle S_{\boldsymbol{k}}^{+} \mid S_{\boldsymbol{q}}^{-}\right\rangle\right\rangle_{\omega}$, then we obtain an expression for the Green function $\left\langle\left\langle a_{\boldsymbol{k}, \uparrow}^{+} a_{\boldsymbol{p}, \downarrow} \mid m_{\boldsymbol{q}}^{-}\right\rangle\right\rangle_{\omega}$

$$
\begin{gathered}
\left\langle\left\langle a_{\boldsymbol{k}, \uparrow}^{+} a_{\boldsymbol{p}, \downarrow} \mid m_{\boldsymbol{q}}^{-}\right\rangle\right\rangle_{\omega}=\frac{\delta_{\boldsymbol{p}-\boldsymbol{k}, \boldsymbol{q}}}{2 \pi \sqrt{N}} \frac{f_{k}^{\uparrow}-f_{p}^{\downarrow}-2 U m A(\boldsymbol{p}-\boldsymbol{k} \mid \omega)}{\omega-\mu_{\mathrm{B}} h+I M-2 U m-t_{p}+t_{k}}+ \\
+\frac{I}{2 \sqrt{N}} \frac{f_{k}^{\uparrow}-f_{p}^{\downarrow}-2 U m A(\boldsymbol{p}-\boldsymbol{k} \mid \omega)}{\omega-\mu_{\mathrm{B}} h+I M-2 U m-t_{p}+t_{k}}\left\langle\left\langle S_{\boldsymbol{p}-\boldsymbol{k}}^{+} \mid m_{\boldsymbol{q}}^{-}\right\rangle\right\rangle_{\omega} .
\end{gathered}
$$

The function $A(\boldsymbol{p}-\boldsymbol{k} \mid \omega)$ is defined by equation (40). From equation (16) in the momentum space we obtain

$$
\left\langle\left\langle S_{\boldsymbol{k}}^{+} \mid m_{\boldsymbol{q}}^{-}\right\rangle\right\rangle_{\omega}=\frac{I M}{\omega-g \mu_{\mathrm{B}} h+I m-M\left(J_{0}-J_{k}\right)}\left\langle\left\langle m_{\boldsymbol{k}}^{+} \mid m_{\boldsymbol{q}}^{-}\right\rangle\right\rangle_{\omega} .
$$

Now from equation (32) let us obtain the final expression for the Green function

$$
\left\langle\left\langle m_{\boldsymbol{k}}^{+} \mid m_{\boldsymbol{q}}^{-}\right\rangle\right\rangle_{\omega}=\frac{\delta_{\boldsymbol{k}, q}}{2 \pi} \frac{2 m+F(\boldsymbol{k} \mid \omega)}{\omega-\mu_{\mathrm{B}} h+G(\boldsymbol{k} \mid \omega)} .
$$

Here we use the notations

$$
F(\boldsymbol{k} \mid \omega)=\frac{1}{N} \sum_{\boldsymbol{p}} \frac{\left(t_{\boldsymbol{p}}-t_{\boldsymbol{p}-k}\right)\left(f_{\boldsymbol{p}-k}^{\uparrow}-f_{\boldsymbol{p}}^{\downarrow}-2 U m A(\boldsymbol{k} \mid \omega)\right)}{\omega-\mu_{\mathrm{B}} h+I M-2 U m-t_{\boldsymbol{p}}+t_{\boldsymbol{p}-k}},
$$


and function $G(\boldsymbol{k} \mid \omega)$ in the Green function denominator is

$$
\begin{gathered}
G(\boldsymbol{k} \mid \omega)=\frac{I M\left(\omega-g \mu_{\mathrm{B}} h\right)}{\omega-g \mu_{\mathrm{B}} h+I m}-\frac{m I^{2} M^{2}\left(J_{0}-J_{k}\right)}{\left(\omega-g \mu_{\mathrm{B}} h+I m\right)^{2}}- \\
-\frac{I^{2} M}{2} \frac{F(\boldsymbol{k} \mid \omega)}{\omega-g \mu_{\mathrm{B}} h+I m-M\left(J_{0}-J_{k}\right)} .
\end{gathered}
$$

Equation (50) can be rewritten as

$$
\begin{gathered}
F(\boldsymbol{k} \mid \omega)=-\frac{1}{N} \sum_{\boldsymbol{p}}\left(f_{\boldsymbol{p}}^{\uparrow}-f_{\boldsymbol{p}+\boldsymbol{k}}^{\downarrow}\right)+\left(\omega+\Delta_{0}\right) \chi_{0}^{+-}(\boldsymbol{k}, \omega)- \\
-2 U m \chi_{0}^{+-}(\boldsymbol{k}, \omega)\left[\frac{1}{N} \sum_{\boldsymbol{p}} \frac{\omega+\Delta_{0}}{\omega-E_{\boldsymbol{p}+\boldsymbol{k}, \downarrow}^{0}+E_{\boldsymbol{p}, \uparrow}^{0}}-1\right] .
\end{gathered}
$$

Here the spectrum of band electrons is expressed by

$$
E_{\boldsymbol{k}, \sigma}^{0}=t_{\boldsymbol{k}}+\frac{U n}{2}+\frac{\sigma}{2}\left(I M-\mu_{\mathrm{B}} h-2 U m\right), \quad \sigma= \pm 1 .
$$

From the last relation we can conclude that the electron band spectrum consists of two subbands which correspond to spin-polarized states of electrons. This is a well-known fact in the theory of $s-d$ models of metal magnetism $[1,2],[10,11]$. The Stoner gap that characterizes a shift of one subband with respect to the others is given by the formula

$$
\Delta=I M-2 U m-\mu_{\mathrm{B}} h,
$$

and $\Delta_{0}=I M-2 U m$ is the Stoner gap in the absence of the external magnetic field.

In formula (53) the term $U n / 2(n-$ is the number of band electrons per one atom of metal) describes the shift of electron energy of both Stoner subbands to the Hartree-Fock energy.

\subsection{Spectrum}

The Green functions poles determine the energy of magnon excitations in the system. From the requirement $R(\boldsymbol{k}, \omega)=0$, when $h \equiv 0$, we find an equation for the spin excitation spectrum:

$$
\begin{gathered}
{\left[\omega-M\left(J_{0}-J_{k}\right)\right][\omega+I M]+I m \omega+} \\
+\frac{I^{2} M}{2}\left[\frac{1}{N} \sum_{\boldsymbol{p}}\left(f_{\boldsymbol{p}}^{\uparrow}-f_{\boldsymbol{p}+\boldsymbol{k}}^{\downarrow}\right)-\left(\omega+\Delta_{0}\right) \chi_{0}^{+-}(\boldsymbol{k}, \omega)\right]+ \\
+I^{2} U M m \chi_{0}^{+-}(\boldsymbol{k}, \omega)\left[\frac{1}{N} \sum_{\boldsymbol{p}} \frac{\omega+\Delta_{0}}{\omega-E_{\boldsymbol{p}+\boldsymbol{k}, \downarrow}^{0}+E_{\boldsymbol{p}, \uparrow}^{0}}-1\right]=0 .
\end{gathered}
$$


Relation (55) is a rather complicated transcendent equation with respect to $\omega$. However, while solving it one must take into account the assumptions made while calculating the Green functions. In particular, equation (55) is valid in the longrange limit $\boldsymbol{k} \rightarrow 0$, which pertains to the Tyablikov-like decoupling.

For the wave vector $\boldsymbol{k}=0$ the solution of equation (55) consists of two roots:

$$
\omega_{0}^{+} \equiv \omega^{+}(\boldsymbol{k}=0)=0 ; \quad \omega_{0}^{-} \equiv \omega^{-}(\boldsymbol{k}=0)=-I(m+M) .
$$

Thus, for small wave vector values there are two branches of spin excitation: one is of an "optical" and the other of an "acoustic" type. The dispersion of the acoustic branch in the long-wave region is determined from the transcendental equation

$$
\begin{gathered}
\omega^{+}=\frac{M^{2}}{m+M}\left(J_{0}-J_{\boldsymbol{k}}\right)-\frac{I M}{2(m+M)}\left[\frac{1}{N} \sum_{\boldsymbol{p}}\left(f_{\boldsymbol{p}}^{\uparrow}-f_{\boldsymbol{p}+\boldsymbol{k}}^{\downarrow}\right)-\left(\omega^{+}+\Delta_{0}\right) \chi_{0}^{+-}\left(\boldsymbol{k}, \omega^{+}\right)\right]- \\
-\frac{I M U m}{m+M} \chi_{0}^{+-}(\boldsymbol{k}, \omega)\left[\frac{1}{N} \sum_{\boldsymbol{p}} \frac{\omega^{+}+\Delta_{0}}{\omega^{+}-E_{\boldsymbol{p}+\boldsymbol{k}, \downarrow}+E_{\boldsymbol{p}, \uparrow}^{0}}-1\right]
\end{gathered}
$$

and for the optical branch-from the following equation:

$$
\begin{gathered}
\omega^{-}=-I(m+M)+\frac{m M}{m+M}\left(J_{0}-J_{\boldsymbol{k}}\right)+ \\
+\frac{I M}{2(m+M)}\left[\frac{1}{N} \sum_{\boldsymbol{p}}\left(f_{\boldsymbol{p}}^{\uparrow}-f_{\boldsymbol{p}+\boldsymbol{k}}^{\downarrow}\right)-\left(\omega^{-}+\Delta_{0}\right) \chi_{0}^{+-}\left(\boldsymbol{k}, \omega^{-}\right)\right]+ \\
+\frac{I M U m}{m+M} \chi_{0}^{+-}\left(\boldsymbol{k}, \omega^{-}\right)\left[\frac{1}{N} \sum_{\boldsymbol{p}} \frac{\omega^{-}+\Delta_{0}}{\omega^{-}-E_{\boldsymbol{p}+\boldsymbol{k}, \downarrow}+E_{\boldsymbol{p}, \uparrow}^{0}}-1\right] .
\end{gathered}
$$

When we have an identity to zero of the following values $J_{\boldsymbol{k}} \equiv 0$ and $U \equiv 0$, which indicates the absence of direct spin-spin and Coulomb interactions, it is possible to receive a dispersing equation for an ordinary $s-d$-model.

$$
\begin{gathered}
\omega^{+}=-\frac{I M}{2(m+M)} \frac{1}{N} \sum_{\boldsymbol{p}} \frac{\left(t_{\boldsymbol{p}+\boldsymbol{k}}-t_{\boldsymbol{p}}\right)\left(f_{\boldsymbol{p}}^{\uparrow}-f_{\boldsymbol{p}+\boldsymbol{k}}^{\downarrow}\right)}{\omega^{+}-E_{\boldsymbol{p}+\boldsymbol{k}, \downarrow}^{0}+E_{\boldsymbol{p}, \uparrow}^{0}} \\
\omega^{-}=-I(m+M)+\frac{I M}{2(m+M)} \frac{1}{N} \sum_{\boldsymbol{p}} \frac{\left(t_{\boldsymbol{p}+\boldsymbol{k}}-t_{\boldsymbol{p}}\right)\left(f_{\boldsymbol{p}}^{\uparrow}-f_{\boldsymbol{p}+\boldsymbol{k}}^{\downarrow}\right)}{\omega^{-}-E_{\boldsymbol{p}+\boldsymbol{k}, \downarrow}^{0}+E_{\boldsymbol{p}, \uparrow}^{0}}
\end{gathered}
$$

The following equations (59-60) have a different functional form in comparison with equation [17] because in calculations of the spectrum another approximation was used.

For the small values of wave vector $\boldsymbol{k}$ the magnon dispression law is quadratic:

$$
\omega^{+}(\boldsymbol{k})=\omega_{0}^{+}+\frac{M}{m+M} D_{1} k^{2}-\frac{1}{2} \frac{M}{m+M} \frac{D_{2}^{+}}{\omega_{0}^{+}+\Delta_{0}} k^{2},
$$




$$
\omega^{-}(\boldsymbol{k})=\omega_{0}^{-}+\frac{M}{m+M} D_{1} k^{2}+\frac{1}{2} \frac{M}{m+M} \frac{D_{2}^{-}}{\omega_{0}^{-}+\Delta_{0}} k^{2},
$$

where $D_{1}$ is the spin stifness constant, taking into account only a direct spin-spin interaction. The quantities

$$
D_{2}^{ \pm}=\frac{1}{N} \sum_{\boldsymbol{q}}\left[\frac{1}{2}\left(f_{\boldsymbol{q}}^{\uparrow}+f_{\boldsymbol{q}}^{\downarrow}\right) q^{2}(\boldsymbol{\nabla})^{2} t_{q}+\frac{1}{\omega_{0}^{ \pm}+\Delta_{0}}\left(f_{\boldsymbol{q}}^{\uparrow}-f_{\boldsymbol{q}}^{\downarrow}\right)\left(\boldsymbol{q} \nabla t_{q}\right)^{2}\right]
$$

determine the correction to magnon masses conditioned by spin-electron interactions.

The equation for the spectrum of spin waves can also be derived from the pole of the Green function $\left\langle\left\langle m_{\boldsymbol{k}}^{+} \mid m_{\boldsymbol{q}}^{-}\right\rangle\right\rangle_{\omega}$. From formula (49) the equation follows:

$$
(\omega+I M)\left[\omega+I m-M\left(J_{0}-J_{k}\right)\right]-\frac{I^{2} M}{2} F(\boldsymbol{k} \mid \omega)=0
$$

From this equation, under the long-wave boundary condition, dispersing ratio of the acoustic and optical branches identical to expressions (57)-(58) are obtained.

\section{Conclusions}

The Hartree-Fock and Tyablikov approximations are used for closing the equations of motion for the Green functions, and equations for the spectrum of spin waves of the $s-d$ model are obtained. In the long-wave region there are two branches of magnon excitations. So the spectrum structure of $s-d$ spin-wave excitations is known and described in papers $[1,15-16]$. The physical nature of the two branches of the spectrum indicates, that the $s-d$ model is in some sense equivalent to two-sublattice ferromagnetic. The equations obtained for partial cases yield the results like in the papers mentioned above.

Equations (57)-(58) are correct for both the ferromagnetic type of spin-electron interaction $(J<0)$ and for the antiferromagnetic one $(J>0)$. But in the second case it is necessary to redefine the expression for full magnetization by replacing $M+m$ with $M-|m|$ and the electron spin polarization $m$ with $-|m|$ in the equations.

\section{References}

1. Vonsovsky S.V., Izyumov Yu.A. The electron theory of transient metals. I. // UFN, 1962, 77, No 3, p. 377-448 (in Russian).

2. Vonsovsky S.V., Izyumov Yu.A. The electron theory of transient metals. II. // UFN, 1962, 78, No 1, p. 3-52. (in Russian).

3. Metfessel' Z., Mattis D. Magnetic semiconductors. M., "Mir", 1972 (in Russian).

4. Nagaev E.L. Physics of magnetic semiconducting. M, "Nauka", 1979 (in Russian).

5. Tyablikov S.V. Qquantum theory magnetism methods. M, "Nauka", 1975, (in Russian). 
6. Akhiezer A.I., Bar'jahtar V.G., Peletminsky S.V. Spin waves. M, "Nauka" , 1967 (in Russian).

7. Lutovinov V.S., Molodyh O.E., Savchenko M.A. The magnon's contribution to electroresistance of ferromagnetic alloys. // Fiz. Magn. Mat., 1986, vol. 61, No 4, p. 707717 (in Russian).

8. Privorotsky I.A. About a capability superconductivity at antiferromagnetics. // JETF, 1962, vol. 43, No 12, p. 2255-2260 (in Russian).

9. Turov V.A., Petrov M.P. Nuclear magnetic resonance in ferro- and antiferromagnetics. M, "Nauka", 1969 (in Russian).

10. Vonsovsky S.V., Izyumov Yu.A. Statistical properties of an electron system of ferromagnetic transient metals. // Fiz. Magn. Mat., 1960, vol. 10, No 3, p. 321-335 (in Russian).

11. Izjumov Yu.A., Kassan-ogly F.A., Skryabin Yu.I. Field methods in theory ferromagnetisms. M, "Nauka", 1969 (in Russian).

12. Leftoulov B.M., Berdyshev A.A. Spin waves in ferromagnetic semiconductors. FSS, 1975, vol. 3, No 7, p. 2049-2053 (in Russian).

13. Eruhimov M.Sh., Ovchinnikov S.G. Elementary energizations in anisotropic narrow band semiconductors. // Teor. Mat. Fiz., 1986, vol. 67, No 2, p. 237-251 (in Russian).

14. Didukh L.D., Stasyuk I.V. Spin-wave consideration of ferromagnetism at the registration of polar energizations. // Ukr.Fiz. Zhurn., 1968, No 6, p. 1924-1926 (in Russian).

15. Berdyshev A.A., Leftoulov B.M. Ferromagnetic metal generalized models. // Fiz. Magn. Mat., 1974, vol. 37, No 2, p. 427-429 (in Russian).

16. Irkhin V.Yu., Katsnelson M.I. Spin waves in narrow band semiconductors. // JETP, 1985, vol. 85, No 2, p. 522-531 (in Russian).

17. Irkhin V.Yu., Kantsnelson M.I. Spin waves in narrow band ferromagnets. // J. Phys. C: Solid State Phys., 1985, 18, p. 4173-4188.

18. Irkhin V.Yu., Kantsnelson M.I. Problem of Condo magnetics. // Fiz. Magn. Mat., 1991, vol. 85, No 2, p. 16-33 (in Russian).

19. Wesselinowa J.M., Dynamic behaviour of the $s-d$ model. // Phys. Stat. Sol. (b), 1983, 120, p. 585-593.

20. Wesselinowa J.M., Some critical properties of the $s-d$ model. // Phys. Stat Sol. (b), 1984, 126, p. 229-233.

21. Wesselinowa J.M., The dielectric function and reflectivity of ferromagnetic semiconductors above $T_{c}$. // J. Phys. C: Solid StatePhys., 1986, 19, p. 4529-4535. 
Довгохвильві спінові збудження кристалічних $s-d$ моделей

\author{
Ю.Рудавський, Г.Понеділок, Л.Дорош
}

Державний університет "Львівська політехніка"

290646 м. Львів-13, вул. С.Бандери, 12

Отримано 6 червня 1998 p.

Досліджуються довгохвильові спінові збудження в кристалічній $s-d$ моделі. В гамільтоніані моделі крім стандартних спін-електронних процесів враховано також обмінні гейзенбергівські взаємодії локалізованих атомних спінів та кулонівські взаємодії між електронами провідності. Використовується розщеплення типу Хартрі-Фока і Тяблікова для замикання ланцюжка рівнянь руху двочасових температурних функцій Гріна. Отримано рівняння для спектру магнонних збуджень.

Ключові слова: магнон, намагніченість, закон дисперсії

PACS: $75.30 . D s, 75.50 . K$ 